\title{
Increased Formation of Fluorescent Lipid- peroxidation Products in Avocado Peels Precedes Other Signs of Ripening
}

\author{
Shimon Meir, Sonia Philosoph-Hadas, Giora Zauberman, Yoram Fuchs, Miriam Akerman, \\ and Nehemia Aharoni \\ .Department of Fruit and Vegetable Storage, Agricultural Research organization, The Volcani \\ Center, Bet Dagan 50250 Israel
}

\begin{abstract}
Additional index words. Persea americana, climacteric, softening
Abstract. Fluorescent products (lipofuscin-like compounds) of lipid peroxidation, which accumulate with age, were extracted from 'Fuerte' avocado (Persea americana Mill.) peels during ripening. Fractionation and analysis of these fluorescent compounds (FCs) was carried out by an improved method, based on separation of FCs from-chlorophyll by Sep-Pak silica cartridges. A sharp rise in FCs content was found 2 days after harvest in avocado fruits stored at 22C, and ethylene enhanced this rise 3-fold on the 4th day. The accumulation of FCs preceded by at leasts days the onset of climacteric ethylene and respiration and by 2 days the decrease in fruit firmness. Moreover, a 6-foId increase in the FCs concentration occurred during 1 to 2 weeks of storage at SC, but the avocado fruits did not show any other detectable signs of ripening. These results suggest that lipid peroxidation may be regarded as one of the earliest detectable processes occurring during fruit ripening. Thus, an increase of FCs in peel may be employed as a horticultural characteristic for estimating initiation of ripening in avocado fruit.
\end{abstract}

Biological membranes are labile to lipid peroxidation because of their high content of phospholipids and polyunsaturated fatty acids (PUFA). Several lines of evidence indicate that free radical-induced lipid peroxidation plays a rode in bringing about many of the deteriorative changes associated with fruit ripening and leaf senescence (Galliard, 1968; Kimura et al., 1982; Thomas, 1986). In this respect, some suggested that fruit ripening may be viewed as an oxidative phenomenon, as indicated by results demonstrating that the onset of ripening correlates with the peroxide content of fruit tissues (Brennan and Frenkel, 1977; Brennan et al., 1979). Positive correlations between fruit senescence and oxidative or lipid degradation processes have been demonstrated during ripening of various fruits, such as avocado, pear, tomato (Brennan and Frenkel, 1977; Brennan et al., 1979) and litchi (Lin et al., 1988), and during aging of potato tubers (Lojkowska and Holubowska, 1989).

The process of lipid peroxidation occurring during plant senescence yields, among other products, the formation of lipofuscin-like FCs (Malshet and Tappel, 1973), via chain reactions consisting of PUFA, lipid hydroperoxides, and malondialdehyde (MDA) (Frankel, 1980; Harwood, 1988; Mudd, 1967). These fluorescent peroxidation products were shown to accumulate or increase with increasing age in various plant tissues and to be positively correlated with lipid peroxidation processes (Maguire and Haard, 1975, 1976; Meir et al., 1991; Pauls and Thompson, 1984; Wilhelm and Wilhelmova, 1981). Quantitative studies of these FCs in climacteric fruits, such as banana and pears, employing a fluorimetric technique, showed an increase in their content during natural and ethylene-induced ripening (Maguire and Haard, 1975, 1976). However, the formation of FCs has not been related to the pattern of changes in fruit ripening characteristics. Recently, we have developed a rapid

Received for publication 2 Oct. 1990. Contribution from the Agricultural Research Organization, The Volcani Center, Bet Dagan, Israel, no. 3099-E, 1990 series. Supported by grant no. US-1525-88 from BARD, The United StatesIsrael Binational Agricultural Research and Development Fund. The cost of publishing this paper was defrayed in part by the payment of page charges. Under postal regulations, this paper therefore must be hereby marked advertisernent solely to indicate this fact. and convenient method for extraction and determination of FCs in green tissues. By employing this method we showed a remarkable increase in FCs content of parsley leaves during natural and ethylene-induced senescence (Meir et al., 1991).

Avocado is a climacteric fruit that does not ripen on the tree, but ethylene enhances its ripening after detachment (Biale, 1960; Eaks, 1966) when the fruit is stored at low (Zauberman and Fuchs, 1973) or high (Zauberman et al., 1988) temperatures. Since ripening is inhibited at low temperatures and lipid peroxidation processes still proceed, it was of interest to study the sequence of occurrence of these two events in avocado fruit. For this purpose, lipid peroxidation, exhibited by increased formation of fluorescent peroxidation products, was estimated by employing the method developed for determination of FCs in green tissues (Meir et al., 1991). The pattern and time of onset of signs of ripening and changes in FCs levels were studied in parallel during natural and ethylene-induced ripening of 'Fuerte' avocado fruit during storage at 5 and $22 \mathrm{C}$.

\section{Materials and Methods}

Plant materials and treatments. Experiments were performed with 'Fuerte' avocado fruit harvested in midseason (October). Fruits were divided into three groups and stored from the day of harvest under the following conditions: a) Control fruits were stored at $22 \mathrm{C}$ and $85 \%$ relative humidity for 12 days until softening; b) ethylene-treated fruits were exposed to $100 \mu$ lethylene/liter for 2 days in a chamber at $22 \mathrm{C}$, and incubated subsequently in air at $22 \mathrm{C}$ for an additional 10 days; and c) fruits were stored at 5C for 14 days. Fruit firmness, respiration rate, ethylene evolution, and FCs content were determined daily with samples of five fruits from each treatment. Where indicated, treatments were carried out in five replicates and the data presented are expressed as means \pm standard error (SE).

Determination of fruit firmness. The firmness (newtons required to penetrate the fruit) of five individual fruits was determined daily until the fruit was soft. We used a 'Chatillon' pressure

Abbreviations: FCs, fluorescent compounds. 
tester, using a conical tip $6.5 \mathrm{~mm}$ in diameter (Fuchs et al., 1986; Zauberman et al., 1988).

Determination of respiration and ethylene. Five fruits from each treatment were sampled daily and their $\mathrm{CO}_{2}$ and ethylene evolution were determined by enclosing each fruit for $1 \mathrm{~h}$ in a 2-liter jar. The determinations of the gases were performed as described by Zauberman et al. (1988). The data obtained with a representative fruit are illustrated.

Extraction and determination of FCs. Fruits tested for firmness were used subsequently for determination of FCs content. Lipofuscin-like fluorescent compounds were extracted from avocado fruit according to the procedure of Fletcher et al. (1973), as modified by Meir et al. (1991). Basically, 2-g samples of tissue, taken either from the peel or the pulp, were placed in a Polytron homogenizer (Kinematic, Luzern, Switzerland) with $20 \mathrm{ml}$ chloroform-methanol $(2: 1, \mathrm{v} / \mathrm{v})$ solution containing 20 mg butylated hydroxy toluene (BHT)/liter, homogenized for 15 see, then warmed for $5 \mathrm{~min}$ at $45 \mathrm{C}$. The homogenate was transferred to a centrifuge tube, mixed thoroughly with $10 \mathrm{ml} 1.5$ $\mathrm{mM} \mathrm{CaCl}_{2}$ (Folch et al., 1957), and centrifuged (10 rein, 10,00O $\times$ $\mathrm{g})$. The chloroform-rich colored phase was processed further for removal of the interfering pigments: A 400- $\mu$ ll aliquot of the chloroform-rich layer [equivalent to $0.06 \mathrm{~g}$ fresh weight $(\mathrm{FW})$ ] was loaded onto a Sep-Pak silica cartridge (Waters Associates, Milford, Mass.), prewashed with $3 \mathrm{ml}$ of analytical chloroform (Frutarom, stabilized with $0.7 \%$ ethanol). When the column was subsequently washed with $6 \mathrm{ml}$ of analytical chloroform, the FCs remained on the column, while the pigments (chlorophylls and carotenoids) were readily removed by the wash solution. The FCs were then eluted with $2.5 \mathrm{ml}$ methanol and the effluent was collected into tubes each containing $20 \mu \mathrm{l} 1 \mathrm{BHT}\left(20 \mathrm{mg} \cdot \mathrm{ml}^{-1}\right.$ methanol). The concentration of FCs in these methanol-eluted fractions was determined by recording their fluorescence spectra and intensity (excitation at 300 and $355 \mathrm{~nm}$, emission at 460 rim), using an Aminco spectrofluorimeter (model SPF-125). Standardization of the instrument was done with a solution of $1 \mu$ gquinine sulfate/liter (Sigma Israel Chemical Co.) dissolved in $0.1 \mathrm{M} \mathrm{H}_{2} \mathrm{SO}_{4}$. Since all experiments were performed with equal quantities of tissue $(0.06 \mathrm{~g} \mathrm{FW})$ and diluted to the same extent, one fluorescence unit was defined as the relative fluorescence measured. Thus, fluorescence intensity was expressed on the basis of units per $0.06 \mathrm{~g} \mathrm{FW}$. Chloroform extracts stored for 2 weeks at $-20 \mathrm{C}$ gave reproducible results $( \pm 2 \%)$.

\section{Results}

The formation of fluorescent peroxidation products in peel and pulp of avocado fruits was examined at three stages of fruit ripening based on fruit firmness defined as: 1) firm $(\geq 105 \mathrm{~N})$ preclimacteric; 2) beginning to soften ( $\geq$ firmness $\geq 65 \mathrm{~N})$, climacteric; 3 ) soft fruits $(\leq 5 \mathrm{~N})$, postclimacteric. The excitation and emission spectra of FCs isolated from peels of preclimacteric, climacteric, and postclimacteric avocado are illustrated in Fig. 1. These spectra all show two excitation maxima at 300 $\mathrm{nm}$ and $355 \mathrm{~nm}$, and one emission maximum at $460 \mathrm{~nm}$. A sharp 6- to 7-fold increase in fluorescence intensity at both excitation maxima was observed in the course of ripening when comparing preclimacteric and postclimacteric fruits (Fig. 1). Since excitation maxima at 350 to $380 \mathrm{~nm}$ were observed previously in lipid extracts of ripening fruits (Maguire and Haard, 1975, 1976) and senescing leaves (Pauls and Thompson, 1984; Wilhelm and Wilhelmova, 1981), further fluorescence results will refer mainly to excitation at $355 \mathrm{~nm}$. The chloroform-soluble fluorescent substances extracted from avocado pulp exhib-

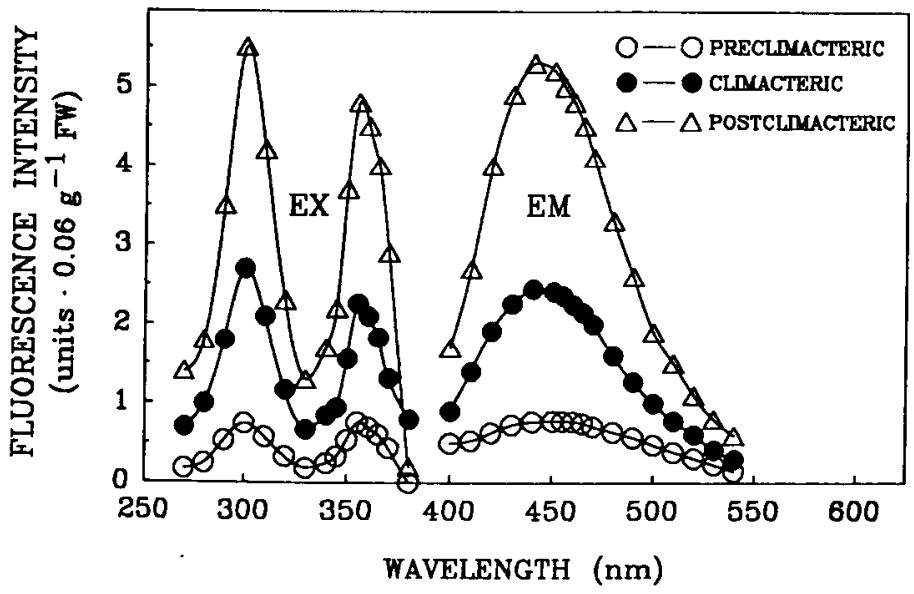

Fig. 1. Representative excitation (EX) and emission (EM) spectra for lipid extracts isolated from peels of control avocado fruits at three ripening stages. The fluorescence intensity of the various extracts was recorded at the indicated wavelengths.

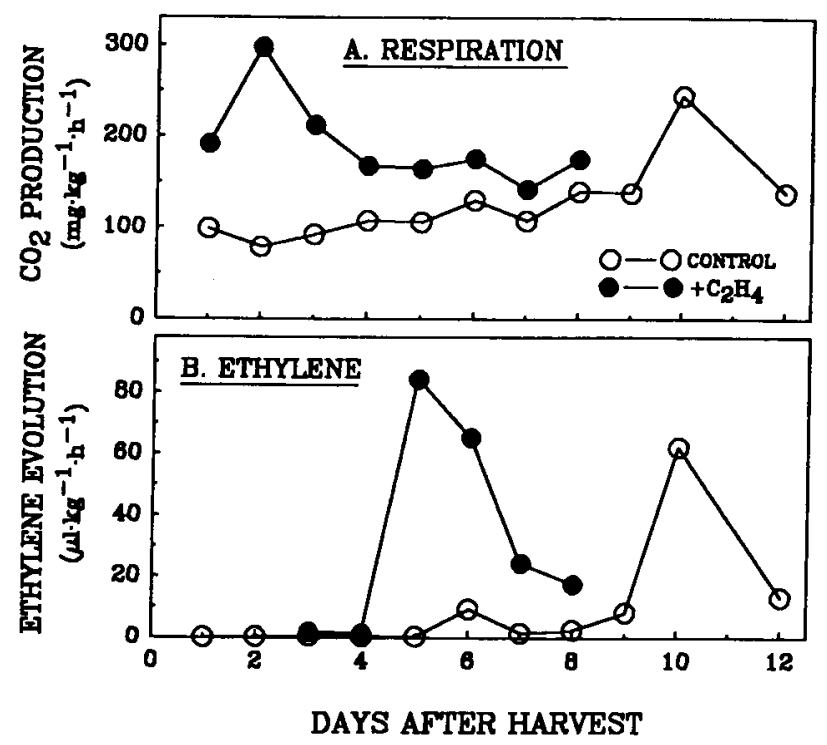

Fig. 2. Effect of exogenous ethylene on $\mathrm{CO}_{2}$ (A) and ethylene evolution (B) during storage of avocado fruits at $22 \mathrm{C}$. 'Fuerte' avocado fruits were exposed to ethylene at $100 \mu l \cdot$ liter $^{-1}$ for 2 days and stored subsequently in air at $22 \mathrm{C}$ for an additional 10 days. Rates of respiration and ethylene evolution of five individual fruits were determined daily, and the data obtained with a representative fruit are illustrated.

ited similar fluorescence spectra, but their content did not increase significantly during ripening (data not shown). Therefore, FCs were extracted in the remaining experiments only from avocado peel.

The pattern of formation of fluorescent peroxidation products was related to the progress of natural and ethylene-induced ripening, the changes and time of onset of signs of ripening, and to FCs content of avocado fruits stored at $22 \mathrm{C}$, with or without ethylene (Fig. 2). The climacteric peaks of both respiration and ethylene appeared in control fruits on the 10th day after harvest. However, exposure of fruits to exogenous ethylene accelerated the respiratory climacteric peak by 8 days (Fig. 2A), and the onset of ethylene production by 5 days (Fig. 2B). The respiratory climacteric in ethylene-treated fruits (Fig. 2A) preceded by 3 days the ethylene climacteric rise (Fig. 2B). A similar delay 
in the appearance of the ethylene climacteric peak following a 24-h ethylene treatment of avocado fruit was observed previously (Zauberman et al., 1988). The corresponding changes in fruit softening and levels of FCs isolated from avocado peel are illustrated in Fig. 3. The decrease in firmness of control fruit (Fig. 3A) preceded by 6 days the climacteric peaks of both respiration and ethylene (Fig. 2). Exposure of fruits to exogenous ethylene accelerated the commencement of fruit softening by 2 days (Fig. 3A). FCs content of control fruits stored at 22C had increased 6 -fold by the 2nd day of storage (Fig. 3B), at which time no measurable changes in fruit firmness (Fig. 3A), respiration rate (Fig. 2A), or ethylene evolution (Fig. 2B) were detected. Thus, this large increase in FCs content of control fruits preceded by 2 days the first detectable changes in fruit firmness and by 8 days the climacteric peaks of respiration and ethylene emanation. The FCs content decreased in ethylenetreated and untreated fruits after 4 or 5 days of storage (Fig. 3B). Nevertheless, after 8 days of storage, the content of FCs in control avocado peels still remained at a level that exceeded by six times their FCs concentration at harvest. Exposure of avocado fruits to ethylene accelerated accumulation of FCs in their peels, relative to the controls, from day 1 to day 4 , showing a peak of fluorescence intensity that was double that of control fruits on day 4 (Fig. 3B). Also, the FCs content of ethylenetreated fruits at day 1 (1.085 units per $0.06 \mathrm{~g} \mathrm{FW})$ was significantly higher $(\mathrm{P} \geq 0.01)$ than that of control fruits $(0.565$ units per $0.06 \mathrm{~g} \mathrm{FW})$ and of that of fruits at harvest $(0.357$ units per 0.06 g FW) (Fig. 3B).

A significant increase in FCs content, ranging from 4- to 9fold, depending on the excitation wavelength, was observed in peels of fruits stored for 1 week at 5C (Table 1). An additional week of storage did not cause any further rise in FCs level. During these storage periods at $5 \mathrm{C}$, no measurable changes in
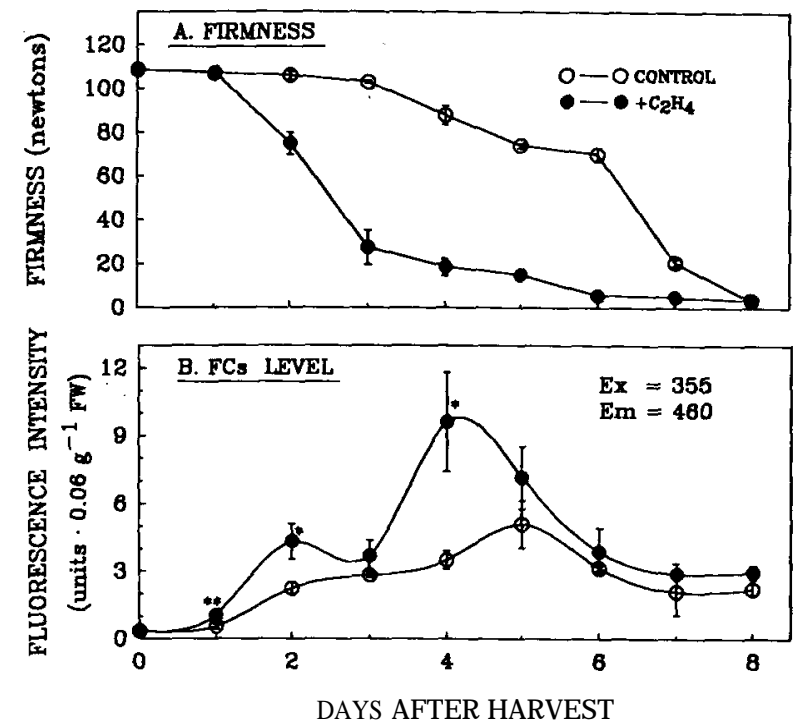

Fig. 3. Effect of exogenous ethylene on firmness (A) and FCs levels (B) of ripening avocado fruits. Fruits were stored as detailed in Fig. 2, and, after being tested for firmness, lipids were extracted from their peels as described in Fig. 1. FCs levels were determined daily by monitoring the fluorescence intensity (excitation, EX, $355 \mathrm{~nm}$; emission, EM, $460 \mathrm{~nm}$ ) of the lipid extracts. The vertical bars represent SE of five replicates. Mean separation by $t$ test between control and treated fruits for each sampling period are indicated by asterisks, when $*$ and $* *$ represent significance at $P=0.05$ and 0.01 , respectively.
Table 1. Effect of cold storage on concentration of lipofuscin-like FCs extracted from peels of 'Fuerte' avocado fruits. ${ }^{2}$

\begin{tabular}{lcr}
\hline $\begin{array}{c}\text { Storage period } \\
\text { at } 5 \mathrm{C} \\
\text { (weeks) }\end{array}$ & \multicolumn{2}{c}{ Fluorescence intensity } \\
\cline { 2 - 3 } & \multicolumn{3}{c}{ Ex 300 nm } & Enits/g fresh wt $355 \mathrm{~nm}$ \\
None (at harvest) & $0.53 \pm 0.03$ & $0.36 \pm 0.01$ \\
1 & $2.00 \pm 0.63$ & $3.30 \pm 1.25$ \\
2 & $1.46 \pm 0.16$ & $2.07 \pm 0.24$ \\
\hline
\end{tabular}

${ }^{2}$ At the indicated periods, the chloroform-soluble lipids were extracted from 2-g peel samples and their fluorescence intensity at $460 \mathrm{~nm}$ was determined after excitation (Ex) at 300 and $355 \mathrm{~nm}$. Results represent mean $\pm \mathrm{SE}$ of five replicates.

the various ripening characteristics of the fruits could be detected (data not shown).

\section{Discussion}

The fluorescence spectra obtained from the lipid extracts of ripening avocado fruits (Fig. 1) were identical to those obtained for FCs extracted similarly from senescing parsley leaves (Meir et al., 1991), and very similar to the spectra measured previously for FCs in ripening pear fruit (Maguire and Haard, 1975), banana pulp and peel (Maguire and Haard, 1976), senescing bean cotyledons (Pauls and Thompson, 1984), and leaves (Wilhelm and Wilhelmova, 1981). There is good evidence that accumulation of these fluorescent peroxidation products may indicate advancement of ripening, since treatment with exogenous ethylene, which is known to enhance avocado ripening (Biale, 1960; Eaks, 1966; Fuchs et al., 1986; Zauberman and Fuchs, 1973; Zauberman et al., 1988), significantly increased the content of such products in avocado peels (Fig. 3B). The increasing fluorescence intensity of lipid extracts with the progress of ripening in avocado peel tissue (Fig. 1) resembled that described for pear fruit (Maguire and Haard, 1975) and banana peel (Maguire and Haard, 1976). Similar to a lack of differences in levels of FCs extracted from avocado pulp, no significant changes in FCs levels could be observed between extracts of preclimacteric and climacteric banana pulp, and only postclimacteric fruits exhibited a certain increase in FCs (Maguire and Haard, 1976). Additionally, similar to data obtained with banana peels (Maguire and Haard, 1976), FCS levels in avocado peel tissue peaked 4 days after ethylene treatment (Fig. 3B). It is noteworthy that a decrease in FCs level following the initial increase was obtained only during fruit ripening (Maguire and Haard, 1975, 1976), whereas leaves accumulated FCs throughout senescence (Meir et al., 1991; Pauls and Thompson, 1984; Wilhelm and Wilhelmova, 1981).

The present study correlates ripening-related changes in FCs level to common signs of ripening, such as fruit firmness and climacteric rises in $\mathrm{CO}_{2}$ and ethylene production. It is clear that the increase in the FCs level (Fig. 3B) preceded by several days the changes in these ripening characteristics (Figs. 2, 3A) during natural and ethylene-induced ripening (except respiration of ethylene-induced fruits). Based on these results and on the previous notion that increased fluorescence of lipid extracts from senescent membranes reflected peroxidative damage (Pauls and Thompson, 1984), we conclude that lipid peroxidation, which involves free radical formation, precedes ripening in avocado fruit.

This conclusion is reinforced by the finding of a remarkable increase in FCs content in peels of avocado fruit that, due to low temperature-storage (Table 1), did not yet exhibit any changes 
associated with ripening. It seems, therefore, that processes of senescence in ripening avocados, and possibly in other fruits, are the consequence of oxidative deterioration of cell membranes, which occurs much earlier than the processes of senescence even at low temperatures (Frankel, 1980). This phenomenon may provide a possible explanation for the observation that the shelf life of avocado fruits transferred from low to room temperature was shorter than that of fruits stored without previous cold storage (Fuchs et al., 1986; Zauberman and Fuchs, 1973). Thus, peroxidative changes occurring in the fruit at low temperature before ripening may be responsible for its enhanced ripening upon transfer to a higher temperature. The possibility of determining the FCs content, one of the earliest detectable processes that occurs during fruit ripening, may be of particular significance with avocado fruit, which do not ripen on the tree. Analysis of the FCs content, as demonstrated here, possibly may be employed as a horticultural tool for estimating the degree of on-tree maturity of avocado fruit.

\section{Literature Cited}

Biale, J.B. 1960. The postharvest biochemistry of tropical and subtropical fruits. Adv. Food Res. 10:293-354.

Brennan, T. and C. Frenkel. 1977. Involvement of hydrogen peroxide in the regulation of senescence in pear. Plant Physiol. 59:411416.

Brennan, T., A. Rychter, and C. Frenkel. 1979. Activity of enzymes involved in the turnover of hydrogen peroxide during fruit senescence. Bet. Gaz. 140:384-388.

Eaks, I.L. 1966. The effect of ethylene upon ripening and respiration rate of avocado fruit. Calif. Avocado Sot. Yrbk. 50:128-133.

Fletcher, B. L., C.J. Dillard, and A.L. Tappel. 1973. Measurement of fluorescent lipid peroxidation products in biological systems and tissues. Anal. Biochem. 52:1-9.

Folch, J., M. Lees, and G.H. Sloane-Stanley. 1957. A simple method for isolation and purification of total lipids from animal tissues. J. Biol. Chem. 226:497-509.

Frankel, E.N. 1980. Lipid oxidation. Progress Lipid Res. 19:1-22.

Fuchs, Y., G. Zauberman, and M. Akerman. 1986. Polygalacturonase, cellulase and softening, as related to ethylene production in avocado fruit stored at chilling temperature. Acta Hort. 179:801-804.

Galliard, T. 1968. Aspects of lipid metabolism in higher plants. II.
The identification and quantitative analysis of lipids from the pulp of pre- and post-climacteric apples. Photochemistry 7:1915-1922.

Harwood, J.L. 1988. Fatty acid metabolism. Annu. Rev. Plant Physiol. Plant Mol. Biol. 39:101-138.

Kimura, S., M. Kanno, Y. Yamada, K. Takahashi, H. Murashige, and T. Okamoto. 1982. The contents of conjugated lipids and fatty acids and the cold tolerance in the mitochondria of Starking Delicious and Rails Janet Apples. Agr. Biol. Chem. 46:2895-2902.

Lin, Z. F., S.S. Li, D.L. Zhang, S.X. Liu, Y.B. Li, G.Z. Lin, and M.D. Chen. 1988. The changes of oxidation and peroxidation in postharvest litchi fruit. Acta Bot. Sinica 30:382-387.

Lojkowska, E. and M. Holubowska. 1989. Changes of the lipid catabolism in potato tubers from cultivars differing in susceptibility to autolysis during the storage. Potato Res. 32:463-470.

Maguire, Y.P. and N.F. Haard. 1975. Fluorescent product accumulation in ripening fruit. Nature (London) 258:599-600.

Maguire, Y.P. and N.F. Haard. 1976. Isolation of lipofuscin-like fluorescent products from ripening banana fruit. J. Food Sci. 41:551554.

Malshet, V.G. and A.L. Tappel. 1973. Fluorescent products of lipid peroxidation: I. Structural requirement for fluorescence in conjugated Schiff's bases. Lipids 8:194-198.

Meir, S., S. Philosoph-Hadas, and N. Aharoni. 1991. Ethylene-increased accumulation of fluorescent lipid-peroxidation products during senescence of parsley leaves, detected by a newly developed method. J. Amer. Soc. Hort. Sci. (In press.)

Mudd, J.B. 1967. Fat metabolism in plants. Annu. Rev. Plant Physiol. 18:229-252.

Pauls, K.P. and J.E. Thompson. 1984. Evidence for the accumulation of peroxidized lipids in membranes of senescing cotyledons. Plant Physiol. 75:1152-1157.

Thomas, H. 1986. The role of polyunsaturated fatty acids in senescence. J. Plant Physiol. 123:97-105.

Wilhelm, J. and N. Wilhelmova. 1981. Accumulation of lipofuscinlike pigments in chloroplasts from senescent leaves of Phaseolus vulgaris. Photosynthetic 15:55-60.

Zauberman, G. and Y. Fuchs. 1973. Ripening processes in avocado stored in ethylene atmosphere in cold storage. J. Amer. Soc. Hort. Sci. 98:477-480.

Zauberman, G., Y. Fuchs, U. Yanko, and M. Akerman. 1988. Response of mature avocado fruit to postharvest ethylene treatment applied immediately after harvest. HortScience 23:588-589. 